\title{
Nodal Solutions for Some Second-Order Semipositone Integral Boundary Value Problems
}

\author{
Huiqin Lu, Yang Wang, and Yansheng Liu \\ School of Mathematical Sciences, Shandong Normal University, Jinan, Shandong 250014, China \\ Correspondence should be addressed to Huiqin Lu; lhy@sdu.edu.cn
}

Received 22 December 2013; Accepted 31 March 2014; Published 27 April 2014

Academic Editor: Gennaro Infante

Copyright (C) 2014 Huiqin Lu et al. This is an open access article distributed under the Creative Commons Attribution License, which permits unrestricted use, distribution, and reproduction in any medium, provided the original work is properly cited.

Using bifurcation techniques, we first prove a global bifurcation theorem for nonlinear second-order semipositone integral boundary value problems. Then the existence and multiplicity of nodal solutions of the above problems are obtained. Finally, an example is worked out to illustrate our main results.

\section{Introduction}

In this paper, we consider the existence and multiplicity of nodal solutions for the following nonlinear second-order semipositone integral boundary value problems (BVP for short):

$$
\begin{gathered}
x^{\prime \prime}(t)+\lambda f(x(t))=0, \quad 0<t<1, \\
x(0)=0, \quad x(1)=\int_{0}^{1} a(s) x(s) d s,
\end{gathered}
$$

where $\lambda>0$ is a parameter, $f \in C(R, R)$, and $a \in L[0,1]$ is nonnegative with $0<\int_{0}^{1} a^{2}(s) d s<1$.

Boundary value problems with integral boundary conditions for ordinary differential equations arise in different areas of applied mathematics and physics. Moreover, they include two, three, multipoint, and nonlocal boundary value problems as special cases. For boundary value problems with integral boundary conditions and comments on their importance, we refer the reader to [1-12] and the references therein.

In [1], utilizing the fixed point index and Leray-Schauder degree theory, Zhang and Sun obtained some existence results for multiple solutions including sign-changing solutions under some technical hypotheses for the following integral boundary value problem:

$$
\begin{gathered}
x^{\prime \prime}(t)+f(x(t))=0, \quad 0<t<1 \\
x(0)=0, \quad x(1)=\int_{0}^{1} a(s) x(s) d s,
\end{gathered}
$$

where $f \in C(R, R), f(0)=0, a \in L[0,1]$ is nonnegative with $\int_{0}^{1} a^{2}(s) d s<1$.

The purpose of this paper is to investigate the existence and multiplicity of sign-changing solutions of BVP (1), having a given number of zeros (so-called "nodal solution"). The existence of such solutions has been investigated for many types of nonlinear Sturm-Liouville problems with separated boundary conditions and multipoint boundary conditions in many recent papers; see [13-18]. Recently, Sun et al. [16] studied for the following $m$-point boundary value problems:

$$
\begin{aligned}
& u^{\prime \prime}(t)+f(u(t))=0, \quad 0<t<1 \\
& u(0)=0, \quad u(1)=\sum_{i=1}^{m-2} \alpha_{i} u\left(\eta_{i}\right),
\end{aligned}
$$

where $\lambda>0$ is a parameter, $f \in C(R, R), f(0)=0$, $\alpha_{i} \in(0,1), i=1,2, \ldots, m-2,0<\sum_{i=1}^{m-2} \alpha_{i}<1$, $0<\eta_{1}<\eta_{2}<\cdots<\eta_{m-2}<1$. By using Rabinowitz's 
global bifurcation theorem, they obtained the existence and multiplicity of nodal solutions when $f_{0} \in(0,+\infty)$, where

$$
f_{0}=\lim _{t \rightarrow 0} \frac{f(t)}{t} .
$$

To the authors' knowledge, there are few papers that have considered the existence of nodal solutions for integral boundary value problems. In [1], Zhang and Sun have obtained signchanging solutions of BVP (2), but no information is obtained regarding the number of zeros of the solution.

Motivated by $[1,15,16]$, in this paper we investigate the existence and multiplicity of nodal solutions for BVP (1). The main features of this paper are as follows. First, the nonlinear term $f$ is semipositone, and $f_{0} \in(0,+\infty)$, where $f_{0}$ is defined as in (4). Next, the methods used here are Rabinowitz's global bifurcation theorem and some of the techniques used in [16], which are entirely different from $[1,7,8]$. Finally, the results we obtained are the existence of at least any given even number of nodal solutions.

Now we give some notations and a global bifurcation theorem which will be used in Section 3. Let $E$ be a real Banach space; Rabinowitz studied a nonlinear eigenvalue problem of the form

$$
u=\lambda L u+H(\lambda, u),
$$

where $\lambda>0$ is a parameter, $L: E \rightarrow E$ is a compact linear map, $H: \varepsilon \equiv R \times E \rightarrow E$ is completely continuous, and $H(\lambda, u)=o(\|u\|)$ for $u$ near 0 uniformly on bounded $\lambda$ intervals. A solution of (5) is a pair $(\lambda, u) \in \varepsilon$ which satisfies (5). The closure of the set of nontrivial solutions of (5) is denoted by $\sum$. If there exist $\mu \in R^{+}=[0,+\infty)$ and $0 \neq v \in E$ such that $v=\mu L v, \mu$ is said to be a positive eigenvalue of $L$ and $v$ is said to be an eigenfunction corresponding to $\mu$. The set of positive eigenvalues of $L$ will be denoted by $r(L)$. The algebraic multiplicity of $\mu \in r(L)$ is $\operatorname{dim} \cup_{j=1}^{\infty} N\left((I-\mu L)^{j}\right)$, where $N(A)$ denotes the null space of $A$. The following was shown in Theorem 1.3 and Theorem 1.25 of Rabinowitz [19] and Theorem 2 of Dancer [20].

Theorem A. If $\mu \in r(L)$ is simple, then $\sum$ possess a maximal subcontinuum $\zeta_{\mu}$ which can be decomposed into two subcontinua $C_{\mu}^{+}$and $C_{\mu}^{-}$such that, for some neighborhood $B$ of $(\mu, 0),(\lambda, u) \in C_{\mu}^{+}\left(C_{\mu}^{-}\right) \cap B$, and $(\lambda, u) \neq(\mu, 0)$ imply $(\lambda, u)=$ $(\lambda, \alpha v+w)$, where $\alpha>0(\alpha<0)$ and $|\lambda-\mu|=o(1)$, $\|w\|=o(|\alpha|)$ for $\alpha$ near 0 . Moreover, either $C_{\mu}^{+}$and $C_{\mu}^{-}$are both unbounded or $C_{\mu}^{+} \cap C_{\mu}^{-} \neq\{(\mu, 0)\}$.

This paper is arranged as the follows: some preliminaries and some lemmas are given including the study of the eigenvalues and eigenfunctions of the linearization of BVP (1) in Section 2. The main results are proved by using Theorem A in Section 3. A concrete example is given to illustrate the application of the main results in Section 4.

\section{Some Preliminaries and Lemmas}

Let $X=C[0,1]$ with the norm $\|x\|=\max _{t \in[0,1]}|x(t)|, Y=$ $\left\{x \in C^{1}[0,1], x(0)=0, x(1)=\int_{0}^{1} a(s) x(s) d s\right\}$ with the norm $\|x\|_{1}=\max \left\{\|x\|,\left\|x^{\prime}\right\|\right\}, Z=\left\{x \in C^{2}[0,1], x(0)=0, x(1)=\right.$ $\left.\int_{0}^{1} a(s) x(s) d s\right\}$ with the norm $\|x\|_{2}=\max \left\{\|x\|,\left\|x^{\prime}\right\|,\left\|x^{\prime \prime}\right\|\right\}$. Then $X, Y, Z$ are Banach spaces.

For any $C^{1}$ function $x$, if $x\left(t_{0}\right)=0$, then $t_{0}$ is said to be a simple zero of $x$ if $x^{\prime}\left(t_{0}\right) \neq 0$. For any integer $k \geq 1$ and any $v \in\{ \pm\}$, as in [15], we define sets $T_{k}^{v} \subset Z$ consisting of the set of functions $x \in Z$ satisfying the following conditions:

(i) $x(0)=0, v x^{\prime}(0)>0$, and $x^{\prime}(1) \neq 0$;

(ii) $x^{\prime}$ has only simple zeros in $(0,1)$ and has exactly $k$ such zeros;

(iii) $x$ has a zero strictly between each two consecutive zeros of $x^{\prime}$.

Note that $T_{k}^{-}=-T_{k}^{+}$and let $T_{k}=T_{k}^{+} \cup T_{k}^{-}$. It is easy to see that the sets $T_{k}^{+}$and $T_{k}^{-}$are disjoint and open in $Z$. Let $E=$ $R \times Y$ under the product topology, $\Phi_{k}^{+}=R \times T_{k}^{+}, \Phi_{k}^{-}=R \times T_{k}^{-}$, and $\Phi_{k}=R \times T_{k}$.

In the following, we give some information on the spectrum structure of the linear integral boundary value problem corresponding to BVP (1):

$$
\begin{gathered}
x^{\prime \prime}(t)+\lambda x(t)=0, \quad 0<t<1 \\
x(0)=0, \quad x(1)=\int_{0}^{1} a(s) x(s) d s .
\end{gathered}
$$

Define the operators $K$ on $Y$ by

$$
(K x)(t)=\int_{0}^{1} k(t, s) x(s) d s,
$$

where

$$
\begin{gathered}
k(t, s)=G(t, s)+\frac{t \int_{0}^{1} G(\tau, s) a(\tau) d \tau}{1-\int_{0}^{1} s a(s) d s}, \\
G(t, s)= \begin{cases}t(1-s), & 0 \leq t \leq s \leq 1 \\
s(1-t), & 0 \leq s \leq t \leq 1 .\end{cases}
\end{gathered}
$$

It is easy to prove the following lemma.

Lemma 1. The linear operator $K: Y \rightarrow Y$ is completely continuous. Moreover, $(\lambda, x) \in(0, \infty) \times C^{2}[0,1]$ is a solution of (6) if and only if $(\lambda, x) \in E$ is a solution of the operator equation $x=\lambda K x$.

We now define a function $G:(0,+\infty) \rightarrow R$ by

$$
G(\eta)=\sin \eta-\int_{0}^{1} a(s) \sin (\eta s) d s, \quad \eta \in(0,+\infty) .
$$

Lemma 2. All the zeros of $G(\eta)$ are simple.

Proof. Suppose that $\eta$ is a double zero of $G(\eta)$; that is,

$$
\sin \eta=\int_{0}^{1} a(s) \sin (\eta s) d s, \quad \cos \eta=\int_{0}^{1} s a(s) \cos (\eta s) d s .
$$


Hence,

$$
\begin{aligned}
1 & =\left(\int_{0}^{1} a(s) \sin (\eta s) d s\right)^{2}+\left(\int_{0}^{1} s a(s) \cos (\eta s) d s\right)^{2} \\
\leq & \int_{0}^{1} a^{2}(s) d s \int_{0}^{1} \sin ^{2}(\eta s) d s \\
& \quad+\int_{0}^{1} s^{2} a^{2}(s) d s \int_{0}^{1} \cos ^{2}(\eta s) d s \\
\leq & \int_{0}^{1} a^{2}(s) d s<1
\end{aligned}
$$

which shows that (10) cannot hold, and so $G(\eta)$ has only simple zeros.

Lemma 3. Suppose that $a(s)$ is symmetrical in $[0,1]$. Then $G(\eta)$ has no zero on $[0, \pi / 2]$ and, for each $i \geq 1, G(\eta)$ has exactly one zero $\eta_{i}=2 i \pi$ on $I_{2 i}:=((2 i-(1 / 2)) \pi,(2 i+(1 / 2)) \pi)$.

Proof. Since $0<\int_{0}^{1} a^{2}(s) d s<1$ and $\int_{0}^{1} a(s) d s \leq$ $\left(\int_{0}^{1} a^{2}(s) d s\right)^{1 / 2}$, we have

$$
0<\int_{0}^{1} a(s) d s<1
$$

Now, (12) implies that $G(\eta)>0$ on $(0, \pi / 2]$; that is, $G(\eta)$ has no zero in this interval, and also

$$
G\left(\left(2 i-\frac{1}{2}\right) \pi\right)<0, \quad G\left(\left(2 i+\frac{1}{2}\right) \pi\right)>0, \quad i \geq 1 .
$$

For each integer $i \geq 1$, by the symmetry of $a(s)$ in $[0,1]$, we have

$$
\begin{aligned}
& \int_{0}^{1} a(s) \sin (2 i \pi s) d s \\
& \quad= \int_{0}^{1} a(1-t) \sin [2 i \pi(1-t)] d t \\
& \quad=-\int_{0}^{1} a(1-t) \sin 2 i \pi t d t \\
& \quad=-\int_{0}^{1} a(t) \sin 2 i \pi t d t
\end{aligned}
$$

So, $2 \int_{0}^{1} a(s) \sin (2 i \pi s) d s=0$; that is, $\int_{0}^{1} a(s) \sin (2 i \pi s) d s=0$.

Thus, $G(2 i \pi)=0$. That is, $G(\eta)$ has one zero $\eta_{i}=2 i \pi$ on each interval $I_{2 i}:=((2 i-(1 / 2)) \pi,(2 i+(1 / 2)) \pi)$.

For any fixed integer $i \geq 1$, suppose that $G(\eta)$ has another zero $\bar{\eta}_{i}$ on $I_{2 i}$. In view of the continuity of $G(\eta)$ and (13), then $G(\eta)$ has the third zero $\tilde{\eta}_{i}$ on $I_{2 i}$. Without loss of generality, we may assume that $\bar{\eta}_{i}<\widetilde{\eta}_{i}$. We have the following three cases to consider. (i) Consider $\bar{\eta}_{i}<\eta_{i}<\tilde{\eta}_{i}$. By (9) and (12), we have

$$
\begin{aligned}
G^{\prime}\left(\eta_{i}\right)=G^{\prime}(2 i \pi) & =\cos (2 i \pi)-\int_{0}^{1} s a(s) \cos (2 i \pi s) d s \\
& =1-\int_{0}^{1} s a(s) \cos (2 i \pi s) d s \\
& >0 .
\end{aligned}
$$

From (13) and Lemma 2, it is easy to see that $G^{\prime}\left(\eta_{i}\right)<0$, which contradicts to (15).

(ii) Consider $\bar{\eta}_{i}<\tilde{\eta}_{i}<\eta_{i}$. From (13) and Lemma 2, it is easy to see that $G^{\prime}\left(\widetilde{\eta}_{i}\right)<0$. So, we have

$$
\begin{gathered}
\sin \left(\widetilde{\eta}_{i}\right)=\int_{0}^{1} a(s) \sin \left(\widetilde{\eta}_{i} s\right) d s, \\
0<\cos \left(\widetilde{\eta}_{i}\right)<\int_{0}^{1} s a(s) \cos \left(\widetilde{\eta}_{i} s\right) d s .
\end{gathered}
$$

Hence,

$$
\begin{gathered}
1<\left(\int_{0}^{1} a(s) \sin \left(\tilde{\eta}_{i} s\right) d s\right)^{2} \\
+\left(\int_{0}^{1} s a(s) \cos \left(\widetilde{\eta}_{i} s\right) d s\right)^{2} \\
\leq \int_{0}^{1} a^{2}(s) d s \int_{0}^{1} \sin ^{2}\left(\widetilde{\eta}_{i} s\right) d s \\
\quad+\int_{0}^{1} s^{2} a^{2}(s) d s \int_{0}^{1} \cos ^{2}\left(\widetilde{\eta}_{i} s\right) d s \\
\leq \int_{0}^{1} a^{2}(s) d s<1,
\end{gathered}
$$

which is a contradiction.

(iii) Consider $\eta_{i}<\bar{\eta}_{i}<\widetilde{\eta}_{i}$. Similar to the proof of Case (ii), we can also lead to a contradiction.

Therefore, $G(\eta)$ has exactly one zero $\eta_{i}=2 i \pi$ on $I_{2 i}:=$ $((2 i-(1 / 2)) \pi,(2 i+(1 / 2)) \pi)$ for each $i \geq 1$.

As the proof of Lemma 4 in [1], it is easy to obtain the following lemma.

Lemma 4. (1) For each $k \geq 1, \eta_{k} \in I_{k}:=((k-(1 / 2)) \pi,(k+$ $(1 / 2)) \pi)$ is one zero of $G(\eta)$ if and only if $\lambda_{k}=\eta_{k}^{2}$ is an eigenvalue of $K$. In addition, $\varphi_{k}(t)=\sin \left(\eta_{k} t\right)$ is an eigenfunction corresponding to $\lambda_{k}$ and $\varphi_{k}(t) \in T_{k}^{+}$.

(2) The algebraic multiplicity of each positive eigenvalue $\lambda_{n}(n=1,2, \ldots)$ of $K$ is 1 .

Lemma 5. Suppose that $a(s)$ is symmetrical in $[0,1]$. Then

(1) there exists a subsequence $\left\{\lambda_{n_{i}}\right\}$ of the eigenvalue sequence $\left\{\lambda_{n}\right\}$ of $K$ such that $\lambda_{n_{i}}=(2 i \pi)^{2}(i=$ $1,2, \ldots)$, and the eigenfunction $\varphi_{n_{i}}$ corresponding to $\lambda_{n_{i}}$ is $\varphi_{n_{i}}(t)=\sin (2 i \pi t)$;

(2) $\varphi_{n_{i}}(t) \in T_{2 i}^{+}$for $i=1,2, \ldots$. 
Proof. From Lemmas 3 and 4, conclusion (1) can be obtained immediately. Noticing that $\varphi_{n_{i}}(t)=\sin (2 i \pi t), i=1,2, \ldots$, it is easy to check that $\varphi_{n_{i}}(t) \in T_{2 i}^{+}$for $i=1,2, \ldots$

Define the operators $F$ and $A$ on $Y$ by

$(F x)(t)=f(x(t))$ and $A x(t)=(K F u)(t)$ for $t \in[0,1]$, respectively, where the operator $K$ is defined as in (7).

It is easy to see that $A: Y \rightarrow Y$ is completely continuous. By direct computation, we can easily get the following lemma.

Lemma 6. $(\lambda, x) \in(0, \infty) \times C^{2}[0,1]$ is a solution of $B V P(1)$ if and only if $(\lambda, x) \in E$ is a solution of equation

$$
x=\lambda A x \text {. }
$$

For $y \in Y$, by the mean-value theorem for the integral, there exists a point $\xi \in(0,1)$ such that

$$
y(1)=\int_{0}^{1} a(s) y(s) d s=y(\xi) \int_{0}^{1} a(s) d s .
$$

Let $x_{0}(t)=1, \omega_{0}(t)=K\left(x_{0}(t)\right)$ for each $t \in[0,1]$ and $e(t)=$ $\xi(1-\xi) t \int_{0}^{1} a(s) d s /\left(1-\xi \int_{0}^{1} a(s) d s\right)$. Let $\omega(t)=M_{0} \omega_{0}(t)$ for each $t \in[0,1]$, where $M_{0}>0$ is a constant to be defined later. The set $W$ is defined by

$$
\begin{aligned}
W=\{x \in X \mid x(t)+\omega(t) & \\
& \geq\|x(t)+\omega(t)\| e(t), t \in[0,1]\} .
\end{aligned}
$$

Obviously $W \in X$ is a closed convex set, and, for each $\tau>0$,

$$
\begin{aligned}
\tau W= & \{y=\tau x \mid x \in W\} \\
= & \left\{y \in X \mid \frac{1}{\tau} y(t)+\omega(t)\right. \\
& \left.\geq\left\|\frac{1}{\tau} y(t)+\omega(t)\right\| e(t), t \in[0,1]\right\} \\
= & \{y \in X \mid y(t)+\tau \omega(t) \\
& \geq\|y(t)+\tau \omega(t)\| e(t), t \in[0,1]\} .
\end{aligned}
$$

Lemma 7. Let $M_{0}$ be a positive number such that $f(x) \geq$ $-M_{0}$, for each $x \in R$. Then

(1) $A: Y \rightarrow W$;

(2) for each $0<\tau_{1}<\tau_{2}<\infty, \tau_{1} W \subset \tau_{2} W$.

Proof. (1) For each $x \in Y$, let $y=A x+\omega=L\left(F x+M_{0} x_{0}\right)$. By direct computation we have

$$
\begin{gathered}
y^{\prime \prime}(t)+\left(f(x(t))+M_{0}\right)=0, \quad 0<t<1 \\
y(0)=0, \quad y(1)=\int_{0}^{1} a(s) y(s) d s .
\end{gathered}
$$

Since $f(x(t))+M_{0} \geq 0$, then $y^{\prime \prime}(t) \leq 0$, and so $y$ is a concave function on $[0,1]$. From (7), it is easy to see that

$$
\begin{aligned}
y(1)= & \frac{1}{1-\int_{0}^{1} s a(s) d s} \\
& \times \iint_{0}^{1} G(\tau, s) a(\tau)\left[f(x(s))+M_{0}\right] d \tau d s \geq 0 .
\end{aligned}
$$

Using the concavity of $y$ and the boundary condition $y(0)=$ $0, y(1) \geq 0$, we can see that $y(t) \geq 0$ for each $t \in[0,1]$ and $\|y\|=\max _{t \in[0,1]} y(t)$; we have from the concavity of $y$ that

$$
y(t) \leq \frac{y(1)-y(\xi)}{1-\xi}(t-1)+y(1), \quad t \in[0, \xi] .
$$

By (19), we have $y(\xi)=y(1) / \int_{0}^{1} a(s) d s$. Hence,

$y(t)$

$$
\begin{aligned}
& \leq y(1) \frac{\int_{0}^{1} a(s)(1-\xi) d s+(1-t)\left(1-\int_{0}^{1} a(s) d s\right)}{\int_{0}^{1} a(s)(1-\xi) d s} \\
& \leq y(1) \frac{\int_{0}^{1} a(s)(1-\xi) d s+\left(1-\int_{0}^{1} a(s) d s\right)}{\int_{0}^{1} a(s)(1-\xi) d s} \\
& =y(1) \frac{1-\xi \int_{0}^{1} a(s) d s}{\int_{0}^{1} a(s)(1-\xi) d s}, \quad t \in[0, \xi] .
\end{aligned}
$$

From the concavity of $y$, we have for each $t \in[\xi, 1]$ that

$$
\begin{aligned}
y(t) & \leq \frac{y(\xi)}{\xi} t \leq \frac{y(\xi)}{\xi} \\
& \leq y(1) \frac{1-\xi \int_{0}^{1} a(s) d s}{\int_{0}^{1} a(s) \xi(1-\xi) d s} .
\end{aligned}
$$

It follows from (25) and (26) that

$$
y(1) \geq \frac{\xi(1-\xi) \int_{0}^{1} a(s) d s}{1-\xi \int_{0}^{1} a(s) d s}\|y\| .
$$

Then we have from the concavity of $y$ that

$$
\begin{aligned}
y(t) & \geq(y(1)-y(0)) t=y(1) t \\
& \geq \frac{\xi(1-\xi) \int_{0}^{1} a(s) d s}{1-\xi \int_{0}^{1} a(s) d s}\|y\| t=\|y\| e(t)
\end{aligned}
$$

that is,

$$
A x(t)+\omega(t) \geq\|A x(t)+\omega(t)\| e(t), \quad t \in[0,1] .
$$

This implies that $A: Y \rightarrow W$, and, therefore, conclusion (1) holds.

(2) Since $A \theta=\theta$, from (1), we see that

$$
\begin{aligned}
\omega(t) & =A \theta(t)+\omega(t) \geq\|A \theta(t)+\omega(t)\| e(t) \\
& =\|\omega(t)\| e(t), \quad t \in[0,1] .
\end{aligned}
$$

For each $x \in \tau_{1} W$, we have

$$
x(t)+\tau_{1} \omega(t) \geq\left\|x(t)+\tau_{1} \omega(t)\right\| e(t), \quad t \in[0,1] .
$$


Then by (30) and (31), we have

$$
\begin{aligned}
x(t)+\tau_{2} \omega(t) & =x(t)+\tau_{1} \omega(t)+\left(\tau_{2}-\tau_{1}\right) \omega(t) \\
\geq & {\left[\left\|x(t)+\tau_{1} \omega(t)\right\|\right.} \\
& \left.\quad+\left(\tau_{2}-\tau_{1}\right)\|\omega(t)\|\right] e(t) \\
\geq & \left\|x(t)+\tau_{2} \omega(t)\right\| e(t), \quad t \in[0,1] .
\end{aligned}
$$

This implies that $x \in \tau_{2} W$. Thus, $\tau_{1} W \subset \tau_{2} W$.

\section{Main Results}

Theorem 8. Suppose that $a(s)$ is symmetrical in $[0,1], f_{0} \in$ $(0, \infty)$, and there exists $M_{0}>0$ such that $f(x) \geq-M_{0}$ for each $x \in R$. Then for each integer $i>0$ and each $v=+$, or - , there exists an unbounded maximal subcontinuum $C_{n_{i}}^{v}$ of solutions of $B V P(1)$ in $\Phi_{2 i}^{v} \cup\left\{\left((2 i \pi)^{2} / f_{0}, 0\right)\right\}$, which meets $\left\{\left((2 i \pi)^{2} / f_{0}, 0\right)\right\}$ in $\sum$ and satisfies

(1) $C_{n_{i}}^{+} \cap(\{\lambda\} \times Y) \neq \emptyset$ for each $i \geq 1, \lambda \geq(2 i \pi)^{2} / f_{0}$;

(2) $C_{n_{i}}^{-} \cap(\{\lambda\} \times Y) \neq \emptyset$ for each $i \geq 1, \lambda \geq(2 i \pi)^{2} / f_{0}$.

Proof. Since $f_{0} \in(0, \infty)$, the operator equation (18) can be rewritten as

$$
x=\lambda f_{0} K x+H(\lambda, x) .
$$

Here $H(\lambda, x)=\lambda A x-\lambda f_{0} K x$ and $K$ is defined as in (7). Obviously, it is easy to see that $H(\lambda, u)=o\left(\|u\|_{1}\right)$ for $u$ near 0 uniformly on bounded $\lambda$ intervals. Notice that $K$ is a compact linear map on $Y$. A solution of $\operatorname{BVP}(1)$ is a pair $(\lambda, x) \in E$. By $f_{0} \in(0, \infty)$, the known curve of solutions $\left\{(\lambda, 0) \mid \lambda \in R^{+}\right\}$ will henceforth be referred to as the trivial solutions. The closure of the set on nontrivial solutions of BVP (1) will be denoted by $\Sigma$ as in Theorem A.

If $H(\lambda, x) \equiv 0$, then (33) becomes a linear system

$$
x=\lambda f_{0} K x .
$$

By Lemmas 3, 4, and 5, (34) possesses an increasing subsequence $\left\{\lambda_{n_{i}} / f_{0}\right\}=\left\{(2 i \pi)^{2} / f_{0}\right\}$ of simple eigenvalues sequence $\left\{\lambda_{n} / f_{0}\right\}$ and $(2 i \pi)^{2} / f_{0} \rightarrow+\infty$ as $i \rightarrow+\infty$. Any eigenfunction $\varphi_{k}(t)=\sin \left(\eta_{k} t\right)$ corresponding to $\lambda_{k} / f_{0}$ is in $T_{k}^{+}$. Moreover, $\varphi_{n_{i}}(t) \in T_{2 i}^{+}$for $i=1,2, \ldots$ and $\varphi_{k}(t) \notin T_{2 i}^{+}$for $k \neq n_{i}$.

Consider (33) as a bifurcation problem from the trivial solution. From Theorem $\mathrm{A}$ and $f_{0} \in(0, \infty)$, it follows that, for each integer $i \geq 1, \sum$ possess a maximal subcontinuum $C_{n_{i}} \subseteq$ $E$ which can be decomposed into two subcontinua $C_{n_{i}}^{+}, C_{n_{i}}^{-}$ such that, for some neighborhood $B$ of $\left((2 i \pi)^{2} / f_{0}, 0\right)$,

$$
(\lambda, x) \in C_{n_{i}}^{+}\left(C_{n_{i}}^{-}\right) \cap B, \quad(\lambda, x) \neq\left(\frac{(2 i \pi)^{2}}{f_{0}}, 0\right),
$$

implying $(\lambda, x)=\left(\lambda, \alpha \varphi_{n_{i}}+w\right)$, where $\alpha>0(\alpha<0)$ and $\mid \lambda-\left((2 i \pi)^{2} / f_{0)} \mid=o(1),\|w\|_{1}=o(|\alpha|)\right.$ for $\alpha$ near 0 .
By (18) and the continuity of the operator $A: Y \rightarrow Z$, the set $C_{n_{i}}^{v}$ lies in $R \times Z$ and the injection $C_{n_{i}}^{\nu} \rightarrow R \times Z$ is continuous. Moreover, note that $C_{n_{i}}^{v} \cap(\{0\} \times Z)=\emptyset$. So, $C_{n_{i}}^{\nu}$ is also a continuum in $R \times Z$, and the above properties hold in $R \times Z$.

Since $T_{2 i}$ is open in $Z$ and $\varphi_{n_{i}}(t) \in T_{2 i}^{+}$, we know that

$$
\frac{x}{\alpha}=\varphi_{n_{i}}+\frac{w}{\alpha} \in T_{2 i}^{+}
$$

for $0 \neq \alpha$ sufficiently small. Then there exists $\epsilon_{0}>0$ such that, for $\epsilon \in\left(0, \epsilon_{0}\right)$, we have

$$
(\lambda, x) \in \Phi_{2 i}, \quad\left(C_{n_{i}} \backslash\left\{\left(\frac{(2 i \pi)^{2}}{f_{0}}, 0\right)\right\}\right) \cap B_{\epsilon} \subset \Phi_{2 i},
$$

where $B_{\epsilon}$ is an open ball in $R \times Z$ of radius $\epsilon$ centered at $\left((2 i \pi)^{2} / f_{0}, 0\right)$. Since $T_{2 i}^{v}$ is open in $Z$, it can follow, similar to the proof of Proposition 4.1 in [15], that

$$
(\lambda, x) \in C_{n_{i}} \cap\left(R \times \partial T_{2 i}\right) \Longrightarrow x=0,
$$

which means $C_{n_{i}} \backslash\left\{\left((2 i \pi)^{2} / f_{0}, 0\right)\right\} \cap \partial \Phi_{2 i}=\emptyset$. Consequently, $C_{n_{i}}$ lies in $\Phi_{2 i} \cup\left\{\left((2 i \pi)^{2} / f_{0}, 0\right)\right\}$.

Similarly we can obtain that $C_{n_{i}}^{v}$ lies in $\Phi_{2 i}^{v} \cup$ $\left\{\left((2 i \pi)^{2} / f_{0}, 0\right)\right\}(\nu=+$ or -$)$. Noticing that $T_{k}^{+} \cap T_{k}^{-}=\emptyset$, it can be obtained that $C_{n_{i}}^{+} \cap C_{n_{i}}^{-}=\left((2 i \pi)^{2} / f_{0}, 0\right)$. From Theorem A, we know that $C_{n_{i}}^{+}$and $C_{n_{i}}^{-}$are unbounded in $R \times Z$.

Let $\lambda \geq(2 i \pi)^{2} / f_{0}$ be fixed. For each $0<\tau<\lambda$ and $x \in Z$, $(\tau, x)$ is a solution of (18), and by Lemma 7, $x=\tau A x \in \tau W \subset$ $\lambda W$. Thus,

$$
x(t)+\lambda \omega(t) \geq\|x(t)+\lambda \omega(t)\| e(t), \quad t \in[0,1],
$$

since

$$
\begin{aligned}
\omega(t) & =M_{0} \omega_{0}(t) \leq M_{0} \frac{t}{1-\xi \int_{0}^{1} a(s) d s} \int_{0}^{1}(1-s) d s \\
& <\frac{\left(M_{0}+1\right) t}{2\left(1-\xi \int_{0}^{1} a(s) d s\right)} \leq c_{1} e(t), \quad t \in[0,1],
\end{aligned}
$$

where $c_{1} \geq\left(M_{0}+1\right) / 2 \xi \int_{0}^{1} a(s)(1-\xi) d s$, and so

$$
x(t) \geq\left(\|x\|-\lambda\|\omega\|-\lambda c_{1}\right) e(t), \quad t \in[0,1] .
$$

Let $R(\lambda)=2\left(\lambda\|\omega\|+\lambda c_{1}\right)$. Then for each $(\tau, x) \in \Sigma, \tau \neq 0$, $\|x\| \geq R(\lambda)$, we have $x(t) \geq 0$ for $t \in[0,1]$. This implies that

$$
\begin{aligned}
& C_{n_{i}}^{+} \cap([0, \lambda] \times\{x \in Y \mid\|x\|=R(\lambda)\})=\emptyset \quad(i \geq 1), \\
& C_{n_{i}}^{-} \cap([0, \lambda] \times\{x \in Y \mid\|x\|=R(\lambda)\})=\emptyset \quad(i \geq 1) .
\end{aligned}
$$

Thus, the conclusion holds and the proof is complete. result.

Immediately, from Theorem 8, we have the following 
Theorem 9. Suppose that all the conditions of Theorem 8 hold. Then, for each $\lambda>(2 i \pi)^{2} / f_{0}, B V P(1)$ has at least $2 i$ nodal solutions $u_{n_{1}}^{+}, u_{n_{1}}^{-}, u_{n_{2}}^{+}, u_{n_{2}}^{-}, \ldots, u_{n_{i}}^{+}, u_{n_{i}}^{-}$in $Y$ such that $u_{n_{i}}^{+}$has $(2 i-1)$ zeros in $(0,1)$ and is positive near $t=0$ and $u_{n_{i}}^{-}$has $(2 i-1)$ zeros in $(0,1)$ and is negative near $t=0$.

\section{An Example}

Consider the following nonlinear second-order integral boundary value problem:

$$
\begin{gathered}
x^{\prime \prime}(t)+f(x(t))=0, \quad 0<t<1 \\
x(0)=0, \quad x(1)=\int_{0}^{1}\left(s-s^{2}\right) x(s) d s,
\end{gathered}
$$

where $f(x)=104 \pi^{2} \sin (x)$.

By direct computation, it is easy to see that $f_{0}=$ $\lim _{x \rightarrow 0}\left(104 \pi^{2} \sin (x) / \sin (x)\right)=104 \pi^{2}$, so, $(2 i \pi)^{2} / f_{0}=$ $4 i^{2} \pi^{2} / 104 \pi^{2}=i^{2} / 26$.

Next, we check that all the conditions of Theorem 9 hold. Take $a(s)=s-s^{2}$. It is clear that $0<\int_{0}^{1} a^{2}(s) d s=1 / 30<$ 1 , and $a(s)$ is symmetrical in $[0,1]$, and $a(s) \in L[0,1]$ is nonnegative. Since $f(x)=104 \pi^{2} \sin (x) \geq 104 \pi^{2}, f_{0}=$ $104 \pi^{2} \in(0, \infty)$, and $\lambda=1$. It follows from Theorem 9 , when $i^{2} / 26<1$, we have $i=1,2,3,4,5$, so the boundary value problem (43) has at least 10 nodal solutions in $C^{1}[0,1]$.

\section{Conflict of Interests}

The authors declare that there is no conflict of interests regarding the publication of this paper.

\section{Acknowledgment}

This research is supported by the Reward Fund for Excellent Young and Middle-Aged Scientists of Shandong Province (BS2011SF022), China.

\section{References}

[1] X. Zhang and J. Sun, "On multiple sign-changing solutions for some second-order integral boundary value problems," Electronic Journal of Qualitative Theory of Differential Equations, no. 44, pp. 1-15, 2010.

[2] M. Benchohra, S. Hamani, and J. Henderson, "Functional differential inclusions with integral boundary conditions," Electronic Journal of Qualitative Theory of Differential Equations, no. 15, pp. 1-13, 2007.

[3] G. L. Karakostas and P. Ch. Tsamatos, "Multiple positive solutions of some Fredholm integral equations arisen from nonlocal boundary-value problems," Electronic Journal of Differential Equations, no. 30, 17 pages, 2002.

[4] G. L. Karakostas and P. Ch. Tsamatos, "Existence of multiple positive solutions for a nonlocal boundary value problem," Topological Methods in Nonlinear Analysis, vol. 19, no. 1, pp. 109121, 2002.
[5] Y. Li and F. Li, "Sign-changing solutions to second-order integral boundary value problems," Nonlinear Analysis, Theory, Methods and Applications, vol. 69, no. 4, pp. 1179-1187, 2008.

[6] A. Lomtatidze and L. Malaguti, "On a nonlocal boundary value problem for second order nonlinear singular differential equations," Georgian Mathematical Journal, vol. 7, no. 1, pp. 133$154,2000$.

[7] J. R. L. Webb and G. Infante, "Positive solutions of nonlocal boundary value problems involving integral conditions," NoDEA. Nonlinear Differential Equations and Applications, vol. 15, no. 1-2, pp. 45-67, 2008.

[8] J. R. L. Webb and G. Infante, "Positive solutions of nonlocal boundary value problems: a unified approach," Journal of the London Mathematical Society. Second Series, vol. 74, no. 3, pp. 673-693, 2006.

[9] Z. Yang, "Existence and nonexistence results for positive solutions of an integral boundary value problem," Nonlinear Analysis: Theory, Methods \& Applications, vol. 65, no. 8, pp. 1489-1511, 2006.

[10] Z. Yang, "Positive solutions of a second-order integral boundary value problem," Journal of Mathematical Analysis and Applications, vol. 321, no. 2, pp. 751-765, 2006.

[11] M. Feng, D. Ji, and W. Ge, "Positive solutions for a class of boundary-value problem with integral boundary conditions in Banach spaces," Journal of Computational and Applied Mathematics, vol. 222, no. 2, pp. 351-363, 2008.

[12] M. Benchohra, J. J. Nieto, and A. Ouahab, "Second-order boundary value problem with integral boundary conditions," Boundary Value Problems, vol. 2011, Article ID 260309, 9 pages, 2011.

[13] R. Ma, "Nodal solutions for a second-order $m$-point boundary value problem," Czechoslovak Mathematical Journal, vol. 56, no. 131, pp. 1243-1263, 2006.

[14] R. Ma and B. Thompson, "Nodal solutions for nonlinear eigenvalue problems," Nonlinear Analysis: Theory, Methods \& Applications, vol. 59, no. 5, pp. 707-718, 2004.

[15] B. P. Rynne, "Spectral properties and nodal solutions for second-order, $m$-point, boundary value problems," Nonlinear Analysis: Theory, Methods \& Applications, vol. 67, no. 12, pp. 3318-3327, 2007.

[16] J. Sun, X. Xu, and D. O'Regan, "Nodal solutions for $m$ point boundary value problems using bifurcation methods," Nonlinear Analysis: Theory, Methods \& Applications, vol. 68, pp. 3034-3046, 2008.

[17] Y. Liu and D. O'Regan, "Some global results using bifurcation methods for second order $m$-point boundary value problems," International Journal of Pure and Applied Mathematics, vol. 81, pp. 251-265, 2012.

[18] Y. Liu and D. O'Regan, "Multiplicity results for a class of fourth order semipositone $m$-point boundary value problems," Applicable Analysis, vol. 91, no. 5, pp. 911-921, 2012.

[19] P. H. Rabinowitz, "Some global results for nonlinear eigenvalue problems," Journal of Functional Analysis, vol. 7, pp. 487-513, 1971.

[20] E. N. Dancer, "On the structure of solutions of non-linear eigenvalue problems," Indiana University Mathematics Journal, vol. 23, pp. 1069-1076, 1974. 


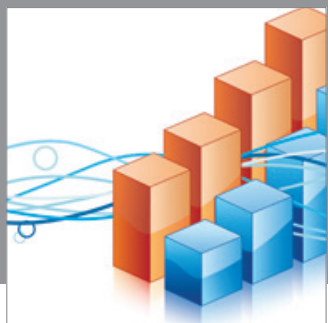

Advances in

Operations Research

mansans

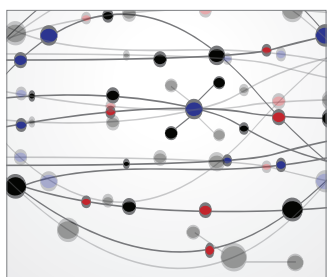

The Scientific World Journal
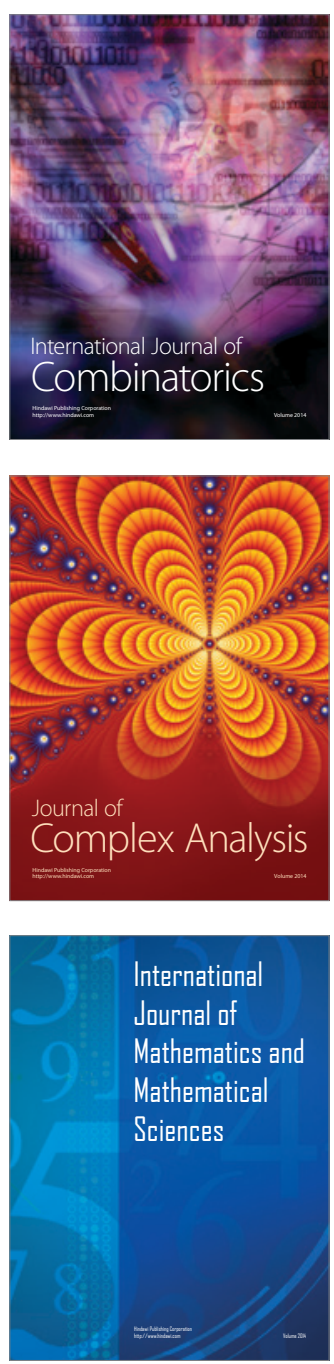
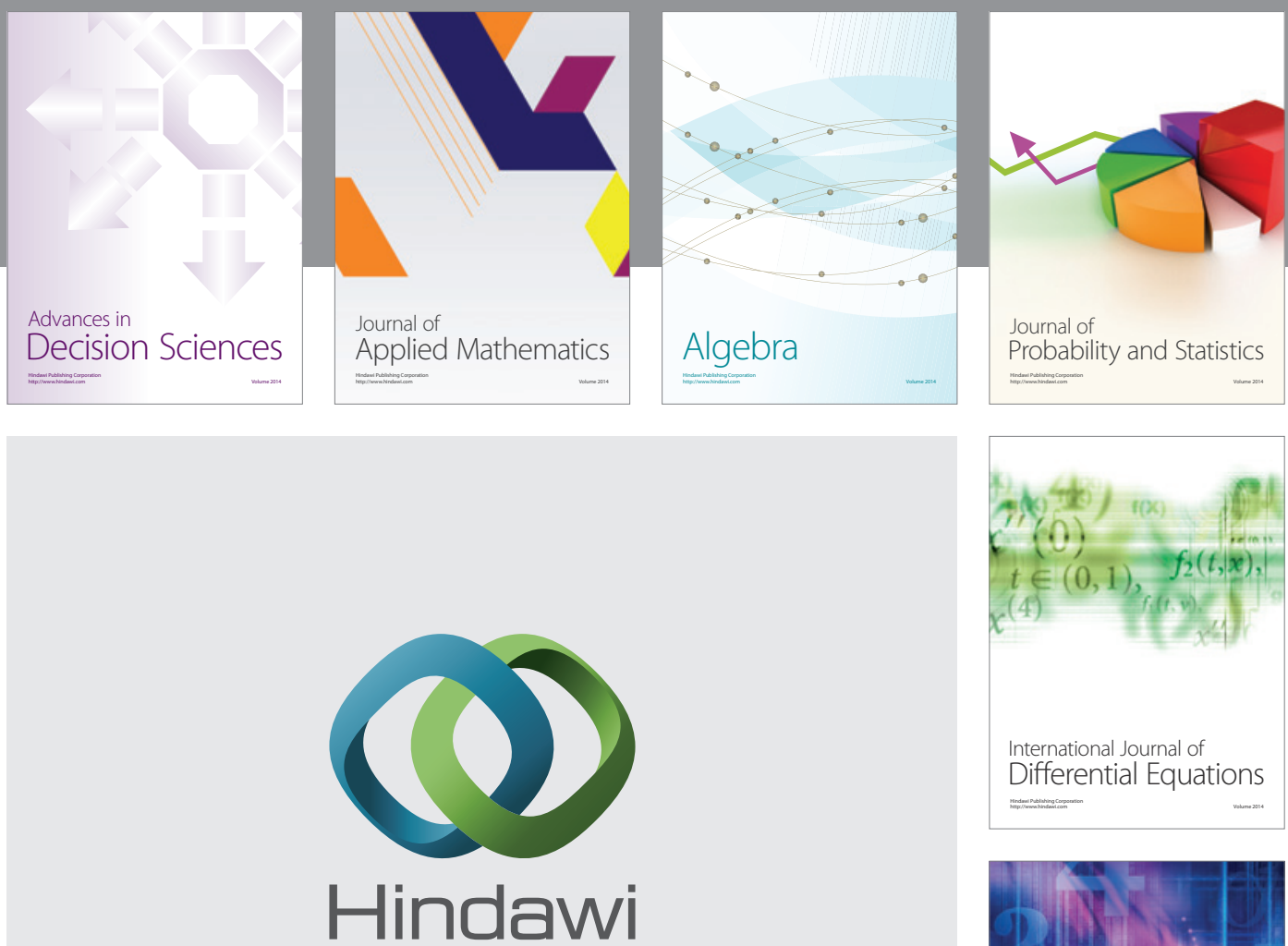

Submit your manuscripts at http://www.hindawi.com
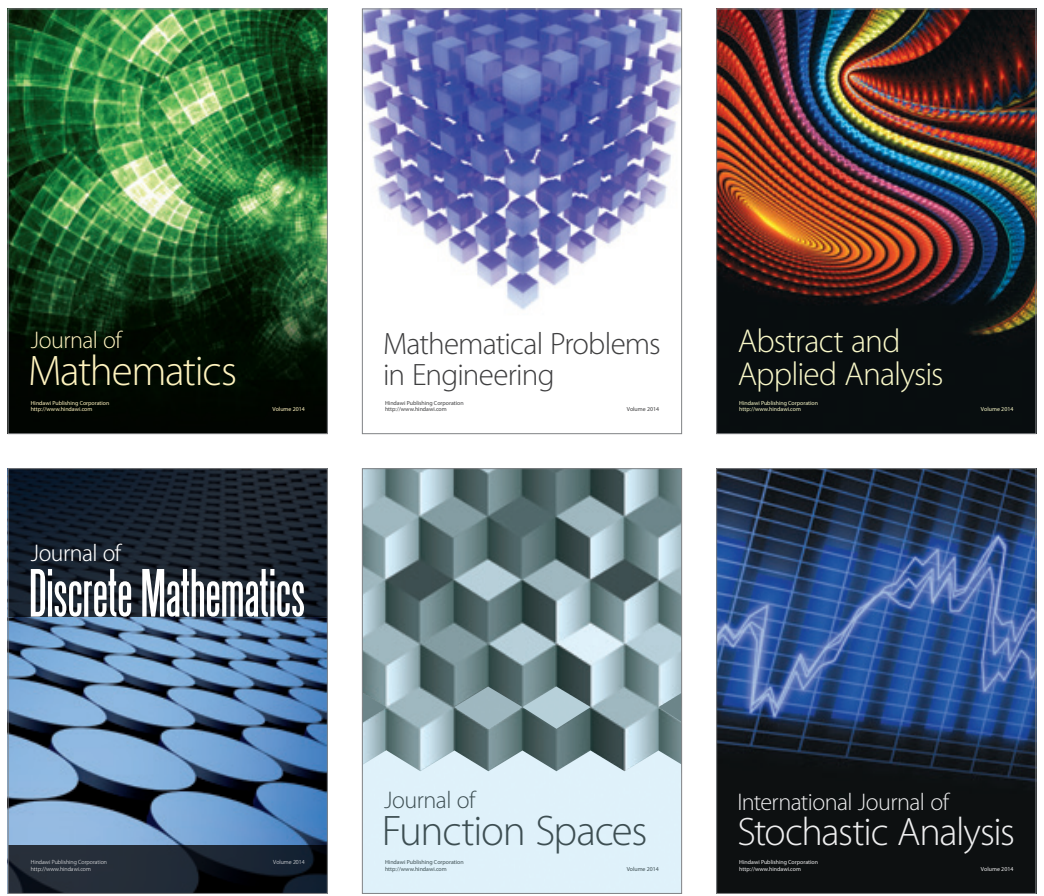

Journal of

Function Spaces

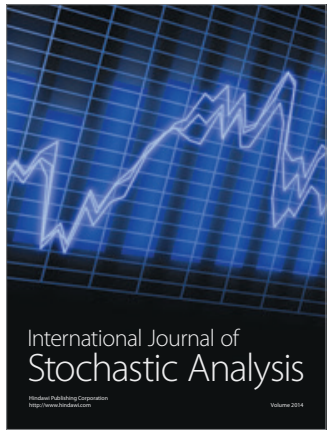

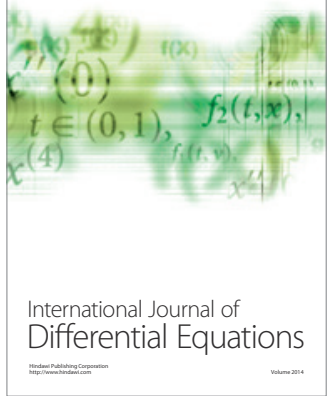
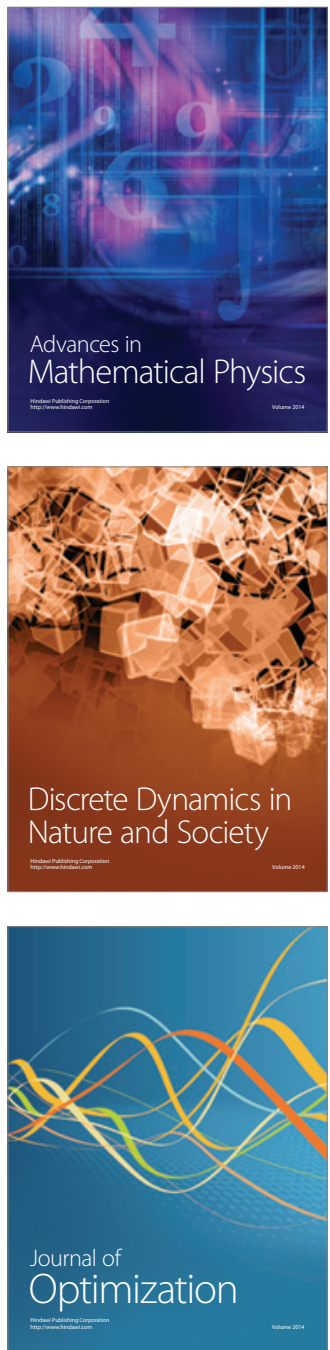